\title{
Digital Assets Price Forecast Based on POW Mining Mechanism
}

\author{
Zhihong Li ${ }^{1}$, Jie Wang1, Kexin $\mathrm{Li}^{2}$ \\ ${ }^{1}$ School of Business Administration, South China University of Technology, Guangzhou, China \\ ${ }^{2}$ School of Journalism and Communication, Shandong University, Shandong, China \\ Email: bmzhhli@scut.edu.cn,793730040@qq.com
}

How to cite this paper: Li, Z.H., Wang, J. and Li, K.X. (2019) Digital Assets Price Forecast Based on POW Mining Mechanism. Open Journal of Social Sciences, 7, 185-198. https://doi.org/10.4236/jss.2019.72016

Received: January 4, 2019

Accepted: February 17, 2019

Published: February 20, 2019

Copyright $\odot 2019$ by author(s) and Scientific Research Publishing Inc. This work is licensed under the Creative Commons Attribution International License (CC BY 4.0).

http://creativecommons.org/licenses/by/4.0/

\begin{abstract}
With the birth of blockchain technology, digital assets such as Bitcoin have also rapidly developed. In recent years, although the development of digital assets has been blocked by national policies, it is still favored by many investors in the capital market. Today, the price of Bitcoin has reached as high as 4000 \$, and some early investors in Bitcoin have received rich returns. But the fluctuations in bitcoin prices are also difficult for investors to ponder, and even let some people lose their money. By analyzing the factors affecting the price of bitcoin, this paper predicts the trend of bitcoin price through linear regression model through the difficulty of mining based on POW mechanism and people's attention to digital assets (Google Trend Index), thus providing investors with some reference comments.
\end{abstract}

\section{Keywords}

Blockchain, Digital Assets, POW, Regression Model

\section{Introduction}

The currency plays an important role in advancing the development of human culture and is a milestone in all stages of human civilization development. The history of currency evolution has gone through five important stages: physical currency, weighing currency, banknotes, electronic money, and digital assets. A person named Satoshi Nakamoto released a blockchain network design called Bitcoin: A Peer-to-Peer Electronic Cash System at the Cryptography Forum in October 31, 2008 [1]. In 2009, the source code of the blockchain technology network was published. And in January 3, 2009, the world's first asset with blockchain technology was born. Despite controversy, blockchain technology is indeed a milestone innovation in Internet technology development. PoW-based 
blockchain digital assets are constantly being solved by miners using computer CPU/GPU computing power, a so-called workload proof mechanism (POW) [2]. After successful cracking, a certain number of digital assets can be obtained as rewards. If you want to calculate a special solution problem, you need to pay the corresponding probability of calculation [3]. Whoever has more computing power, the possibility of calculating the special solution is even greater. Therefore, the use of the POW mechanism to participate in mining will pay amount of computing power. A certain number of special solutions will be set in the background. The number of special solutions set in the background is fixed. Therefore, the number of digital assets in a blockchain network based on the POW mechanism is limited, and the calculation difficulty of the special solution will be continuously improved, so as to control the number of digital assets that can be mined [4]. Similarly, the amount of gold in the earth is also limited.

There are two forms of obtaining digital assets. The first form is based on the POW mechanism, which solves special solutions. The second form is purchased through a trading platform. Cracking special solutions is the competition about computing power. The better the computer performance, the more likely it is to dig into digital assets. However, in today's huge mining market, relying on personal computer mining efficiency is very low [5]. Therefore, in order to increase the mining efficiency, in addition to continuously optimizing the performance of mining equipment, joint mining which called mining pool came into being. The mining is gradually upgraded from the individual to the organization and even the company level. Both parties to a digital asset transaction need a "wallet" and an "address", and the process can be analogized to communication between emails. Both parties to the transaction can use the mobile phone or personal computer to log in to a trading platform to conduct trading. Online purchases on the trading platform are an important channel for acquiring digital assets, and most of the purchase and withdrawal functions can be implemented on the trading platform [6] [7].

\section{Theoretical Background}

The article combines "positivism" and "human behavior" to rationally analyze the market changes of the blockchain digital assets based on the POW mechanism and the factors affecting their prices. First, we conducted an analysis of the price fluctuations of digital assets through surveys, and summarized the main price basis of digital assets in the emerging stage. Secondly, we propose thinking and hypothesis about blockchain digital assets for the current research and market status. Finally, empirical analysis of a large number of data to obtain regression-related conclusions, and test the other digital assets based on the POW mechanism [8].

\subsection{POW Mining Mechanism}

To assess the value of blockchain digital assets, we must mention blockchain 
technology. The essence of the blockchain technology can be traced back to the famous "Byzantine General" issue, the intrinsic meaning of the byzantine general problem is that in the absence of a trusted third party, that is, the authoritative node, how to quickly converge to reach a consensus across the network. Blockchain technology is a good solution to the classic byzantine general problem, reaching no central node, that is, in a distributed situation, the consensus of the whole network is reached [9] [10]. Blockchain technology primarily addresses the issue of reaching consensus in a distributed scenario. The POW mechanism is the main algorithm and means to achieve efficient consensus [11]. The POW mechanism makes blockchain transactions unpredictable and tamper-proof. The core idea of the POW mechanism is to calculate a special solution through the competition of distributed nodes. This special solution is a SHA256 math problem that solves complex difficulties but is easier to verify. The solution to this special solution is the fastest, the miner has the billing rights for the block and will receive a certain amount of bitcoin rewards, and the block is also counted in the longest blockchain [12] [13] [14]. It can be seen that miners participating in the POW mechanism will pay a lot of computing power.

Digital assets based on blockchain technology are gradually becoming familiar to the public. However, in the foreseeable future of blockchain digital assets, it cannot replace the mainstream currency. We assume that the 21 million bitcoin blockchain digital assets can replace the competing currencies and value the digital assets. Comparing cash in circulation (M0) with 21 million bitcoin blockchain digital assets, you can estimate the price of a single digital asset, but in the long run, the valuation of digital assets in this way can only be used as a reference for valuation [15].

Blockchain digital assets are like roller coasters, and the fluctuations are large, which can make people rich overnight, but also make people feel poor. Such large price fluctuations bring great opportunities and challenges to the estimation and forecasting of digital assets [16]. The value of digital assets is difficult to estimate. It is mainly due to the following factors. First of all, the value of digital assets is the recognition price of the digital asset market. Although the mining cost of digital assets is one of the factors, the actual value of the digital assets of the blockchain based on the POW mechanism depends on the mining cost and the combined factor of the digital asset market. Moreover, the quantity of digital asset exploitation in some blockchains is in short supply. From a short-term perspective, the impact of public opinion news on the value of digital assets is far greater than the impact of mining costs on value. Second, blockchain digital assets are new, although gradually recognized by the public, but it is not universally accepted. The skyrocketing and plunging of blockchain digital assets is almost accompanied by hot news in public opinion news, and the introduction of relevant national policies will also affect the price trend of blockchain digital assets, which is difficult to predict [17]. Third, blockchain digital assets have become the new target of the international speculative market is a new prey. As the spe- 
culative capital takes turns, the predictability of its price becomes more difficult [18].

\subsection{Main Factors Affecting the Price of Blockchain Digital Assets}

Blockchain digital asset market is like a roller coaster. We notice that whenever there is a hot event related to blockchain, it will cause the price of this digital asset to skyrocket. According to the analysis of historical price fluctuations in the previous section, and in the development stage of chain digital assets, the following factors are the main factors leading to price fluctuations of blockchain digital assets.

\section{1) Security}

From the events affecting the price fluctuation of digital assets in the blockchain, it can be seen that the security issue is the primary factor affecting the price of digital assets. Hackers attack blockchain networks or trading platform problems. Once such negative events occur, the numbers will be met. Asset prices have a big impact. For example, in June 2011, the hacking incident on the blockchain trading website caused a big sensation in the Bitcoin blockchain network community, which shook the confidence of investors and the price fell. In 2012, Linode, as a web host, lost more than 40,000 bitcoins due to the leak of its server's super-administrative password, causing the price to drop to around $\$ 4$. On February 7, 2014, Mt. Gox suspended the blockchain digital asset redemption business due to technical loopholes, and transaction services were still available, but led to a decline in bitcoin prices. According to the China Information Security Comprehensive Report for the First Half of 2013 issued by the Information Security Research and Service Center of China National Information Center, the primary target of hacker attacks and cyber fraud in China is Bitcoin. Its total number is limited and its value is constantly rising. Due to its high anonymity, it is difficult to trace back once it is stolen. This feature has attracted widespread attention from hackers because of the high level of digital assets in the Bitcoin blockchain. The returnibility and high anonymity that is difficult to trace, make security the primary factor in the price fluctuations of blockchain digital assets.

\section{2) Related policies}

Part of the reason for the price volatility of blockchain digital assets comes from the introduction of relevant policies at the regulatory level in various countries. At the beginning of the development of the blockchain, the regulatory authorities in various countries are still not very clear about the nature and influence of the blockchain network, and there is a lack of supervision. However, with the continuous expansion of the digital asset market in the blockchain in recent years, the influence and transaction volume of digital assets in the blockchain are also increasing. Governments have also attached great importance to the digital assets of the blockchain. Various countries have introduced laws and regulations related to blockchain digital assets, and national policy trends are 
difficult to predict, which will increase the sensitivity to the price of blockchain digital assets. For example, on October 22, 2015, according to the European Court of Justice, digital assets in blockchains in European countries should be treated equally with traditional currencies, and policies to exempt VAT were greatly encouraged, and the supporters of digital assets in the blockchain were greatly encouraged. On January 17, 2018, the price of bitcoin digital assets fell by as much as $25 \%$, because many governments have strengthened the control over the digital assets of the blockchain.

\section{3) Market recognition}

The market's recognition of the value of blockchain digital assets will also affect the price of Bitcoin. For example, from 2009 to 2010, blockchain network technology has just emerged, development is relatively slow, the market is in the early stage of cognition, and the digital asset acceptance of blockchain is not high. In December 2013, Lamborghini dealers announced that Bitcoin blockchain digital assets could be used as payment instruments for Tesla electric vehicles. In 2016, more and more people recognized the value of blockchain digital assets. At the beginning of the year, the People's Bank of China held a seminar on digital currency, aiming to launch a national legal digital currency and discuss the application of digital currency in different scenarios, which greatly inspired Chinese digital currency practitioners and investors. The price of digital assets in the blockchain chain also rose. From the historical market point of view, when the blockchain digital assets or related events are approved by the mainstream market, it will trigger domestic and foreign media to report, which will have a significant upward adjustment of the blockchain digital asset prices. Therefore, the recognition and acceptance of blockchain digital assets will be an important factor driving the price increase of blockchain digital assets.

\section{4) Mining cycle that is halved in four years}

According to the basic supply relationship of economics, if demand increases and supply decreases, prices will inevitably rise. The fact that the number of bitcoin blockchain network mining is halved is a well-known fact. On November 28, 2012, the number of bitcoin blockchain network mining was halved, from 7200 to 3600 , due to supply halving. The quantity is in short supply, which makes the price increase. On July 10,2016, entering the second four-year cycle of production halving, resulting in a shortage of demand and rising prices. Therefore, in the time when the number of mining in the blockchain network is halved, it is worthy of investors' attention. The shortage of output will inevitably lead to an increase in prices.

\section{5) Hard forks and other digital assets}

On August 1, 2017, the Bitcoin cash blockchain network appeared, which is a new digital asset generated by the hard fork of the original chain of the Bitcoin blockchain. During the fork, the bitcoin digital asset market fluctuated and fell. After the end of the fork, the market rebounded in September. The hard fork will cause the blockchain digital asset market to fluctuate and fall in the short term, but the trend seems to be rising in the long run, because the hard fork 
solves the performance problem of the blockchain, and more people will accept the blockchain network before and after the fork. And the holder of the digital asset before the fork can get the same amount of forked assets. The forked digital assets are mirrored from the original blockchain, but have different agreements with the previous ones. The new forked digital assets differentiate the capital flow and market attention, so the price of blockchain digital assets will fall in the short term, due to the dilution of the value. The impact of hard forks on blockchain digital assets is short-term, and its price will rise in the long run. Hard forks can be seen as a slowdown in the price of blockchain digital assets.

Due to the open source nature of the Bitcoin blockchain network technology, there are many other blockchain digital assets issued, such as Ethereum. Its ideas and techniques are derived from Nakamoto's first blockchain network, which is arguably the first blockchain digital asset super-issue. Other digital assets are emerging one after another. The first blockchain digital assets have no absolute technical advantage. Due to its open source nature, the market only sees its high price, mining is difficult, other digital assets are cheaper, and it is easier to speculate. Therefore, the existence of digital assets in other blockchains will certainly share the market and affect the price of Bitcoin blockchain assets.

In summary, whether it is security issues, the introduction of national policies, market acceptance, four-year mining halving, forks and other digital assets, it will cause big reports from domestic and foreign media, and blockchain digital assets will gradually entering the public's field of vision, people's attention to the blockchain network will also increase.

\section{Blockchain Digital Assets Valuation Model}

The previous section analyzed the factors affecting the price of digital assets. Cost, safety, national policy, market acceptance, halving of four-year mining quantities, forks and other digital assets all contribute to the value of digital assets. This chapter will use positivism to analyze data on other factors such as cost and safety, national policy control, and mathematical regression fitting model analysis.

\subsection{Correlation between Mining Calculation Difficulty and Digital Assets Value}

Cost is the primary factor in considering the value of digital assets. The first blockchain digital asset was initially acquired by using a computer to solve the problem of special solutions. Therefore, we can roughly estimate the approximate cost of the production unit's digital assets based on the cost of the miner, the electricity cost of the equipment, and the manpower consumption. The input of mining machine and manpower belongs to fixed consumption, and the electricity of average production unit digital assets is mainly related to the calculation difficulty and self-calculation of cracking special solution, so the calculation difficulty of cracking special solution can be changed. It directly reflects the 
trend of the cost of digital assets in the blockchain. In order to prove whether the blockchain digital asset price has certain correlation with the mining calculation difficulty, we select the bitcoin blockchain network price history data provided by Investing.com and the historical mining calculation difficulty data provided by "Bitfan" between September 21, 2014 and January 7, 2018. The data is in weekly units. As shown in Figure 1, we can see that the price movements are basically the same as the calculation difficulty, and generally show an upward trend.

According to Metcalfe's law, as the number of squares of users increases, the usefulness of the network increases. In other words, the value of the network is $\mathrm{V}=\mathrm{K} \times \mathrm{N}^{2}$ ( $\mathrm{K}$ is the value coefficient, $\mathrm{N}$ is the number of users), and the formula indicates that the value of the network is proportional to the square of the number of connected users. For example, the telephone network is proportional to the square of the number of telephone users. Due to the increasing number and technical advancement of mining equipment in various parts of the world, the number of digital assets in the blockchain that can be excavated by equipment with unit computing capacity has gradually decreased, so the cost of bitcoin is rising rapidly, as shown in Figure 1.

Analogy to Metcalf, we make assumptions and verify that the more miners the network is engaged in mining, the more difficult it is to mine, and the higher the value of the blockchain network. Therefore, we suspect that the square of the difficulty of mining calculation is linear with the price of blockchain digital assets, so we plot the historical price of the bitcoin and the square of the difficulty of historical mining calculation into a scatter plot, as shown in Figure 2. As shown, it can be seen that there is a certain linear relationship between the price and the square of the difficulty of mining calculation, so we perform regression analysis on the two variables to further verify the relationship.

It can be seen from Table 1 that the correlation coefficient Multiple $\mathrm{R}$, which measures the degree of correlation between the price and the square of the difficulty of mining calculation, $\mathrm{R}=0.9526$, indicating that the price is highly correlated with the square of the difficulty of mining calculation, and is positively correlated. The coefficient $\mathrm{R}^{2}=0.9075$, which is a high degree of fitting. The ability to explain the price is $90.75 \%$, and the fitting effect is strong. Adjusted $\mathrm{R}$

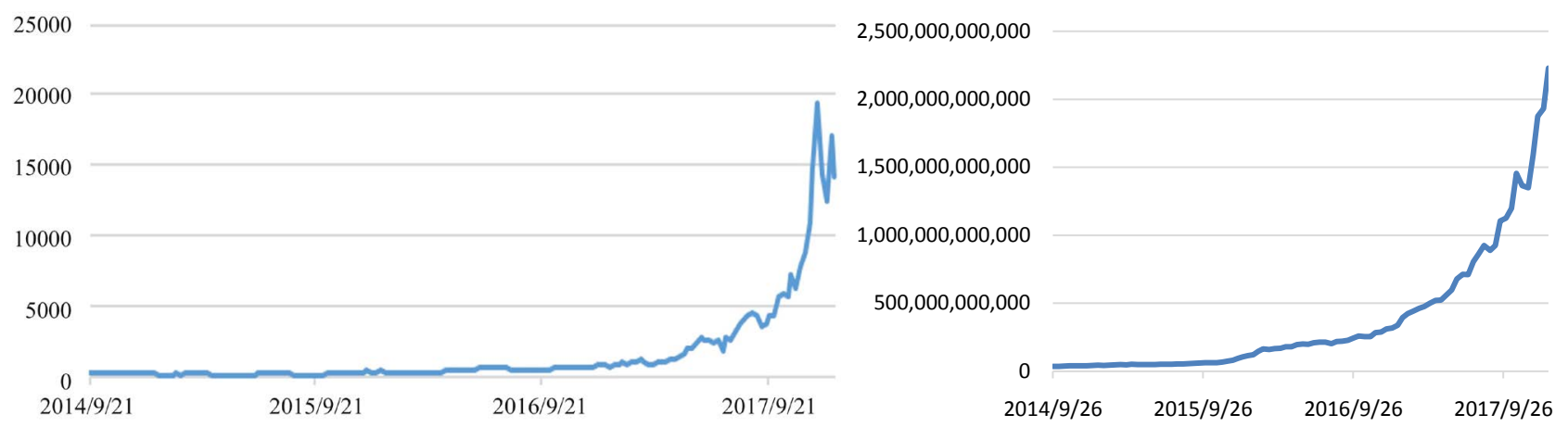

Figure 1. Trends in price and calculation difficulty. 


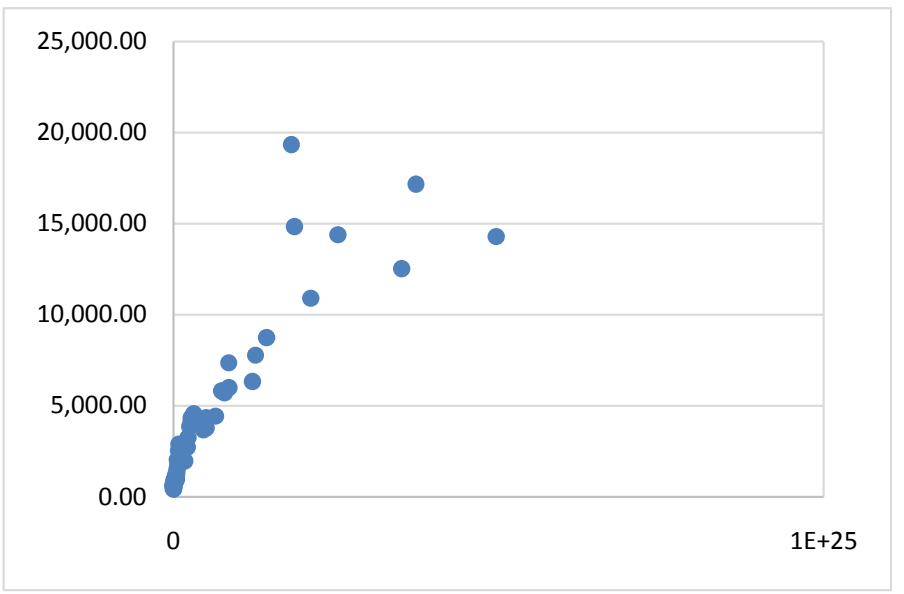

Figure 2. The scatter plot of price and calculation difficulty squared.

Table 1. Topics focus on correlation between volume and issue number.

\begin{tabular}{cc}
\hline & Regression statistics \\
\hline Multiple R & 0.952614598 \\
R Square & 0.907474572 \\
Adjusted R square & 0.906930305 \\
Standard error & 1005.821129 \\
Observations & 172 \\
\hline
\end{tabular}

square $=0.9069$, indicating that the square of the mining calculation difficulty can explain $90.69 \%$ of the price. Table 2 shows the regression effect of the F-significant statistic to determine the price and the mining difficulty square regression model. The $\mathrm{P}$ value in the analysis of the price and mining calculation difficulty is equal to $8.69135 \times 10^{-90}$, which is not significant. The regression effect of the squared regression model of price and mining difficulty is more significant. It can be seen from Table 3 that the $\mathrm{P}$ value of the square of the difficulty of mining calculation is $8.6910 \times 10^{-90}$, which is much smaller than the significance level of 0.05 , indicating that the regression coefficient of the square of mining difficulty is very significant, and the square of mining difficulty is related to price [19] [20] [21] [22].

From the above regression analysis, we prove that the square of the difficulty of mining calculation has a significant linear relationship with the price.

\subsection{Correlation between Search Volume and Price of Digital Prices}

In fact, the rise and fall of blockchain digital assets is formed by cost information combined with market information. In the short-term and medium-term perspectives, the media's positive and bad news has a great impact on digital asset holders or investors. Every time, the blockchain digital assets skyrocketed and plunged almost always accompanied by hot events. These hot events are analyzed in the previous chapter on the behavior of digital asset prices (security, 
Table 2. Variance analysis table of price and calculation difficulty squared.

\begin{tabular}{cccccc}
\hline & df & SS & MS & F & Significance F \\
\hline Regression analysis & 1 & $1.69 \mathrm{E}+09$ & $1.69 \mathrm{E}+09$ & 1667.3 & $8.69 \mathrm{E}-90$ \\
residual & 170 & $1.72 \mathrm{E}+08$ & 1011676 & & \\
total & 171 & $1.86 \mathrm{E}+09$ & & \\
\hline
\end{tabular}

Table 3. Variance regression of price and calculation difficulty squared.

\begin{tabular}{ccccccc}
\hline & Cofficients & Standard error & $\mathrm{t}$ Stat & P value & Lower 95\% & Upper 95\% \\
\hline Intercept & 308.4625 & 84.2117 & 3.6629 & 0.00033 & 142.227 & 474.698 \\
$X$ Variable & $4.75 \mathrm{E}-21$ & $1.16 \mathrm{E}-22$ & 40.833 & $8.69 \mathrm{E}-90$ & $4.52 \mathrm{E}-21$ & $4.98 \mathrm{E}-21$ \\
\hline
\end{tabular}

policy introduction, market acceptance, four-year halving mining rules, hard forks and other digital assets, etc.). According to Robert Shillerr, the trend of blockchain digital asset prices is particularly in line with the definition of speculative bubbles. People are eager to hold digital assets, hoping to gain wealth through value-added, and blockchain digital asset investment is gradually familiar and understood. The most direct response of the news media to the psychological impact of the public can be reflected in the public's attention to this matter. Therefore, we assume that the news media's coverage of the blockchain digital asset will attract public attention, which indirectly will cause changes in the price of digital assets in the blockchain.

Public attention is an abstract concept. Fortunately, the search engine provides us with a lot of quantitative information. The representative Google Trend provides search and click popularity data for specific keywords. So we downloaded Google Trend's search volume data for the keyword "Bitcoin" (in a weekly cycle, standardized $0-100$ numerical scale), which indirectly reflects the value of the Bitcoin blockchain network. We selected the bitcoin price from September 21, 2014 to January 7, 2018 to analyze the search volume of Bitcoin. As shown in Figure 3, we can see that the price trend is basically similar to the search volume of Bitcoin, which is generally on the rise.

It can be seen from Table 4 that the correlation coefficient Multiple R, which measures the correlation between the price and the search volume, $\mathrm{R}=0.9426$, indicates that the price is highly correlated with the search volume and is positively correlated. $\mathrm{R}^{2}=0.8884$, indicating that the search volume has a capacity to explain the price of $88.84 \%$, and the fitting effect is strong. Adjusted $\mathrm{R}^{2}=0.8877$, indicating that the search volume can account for $88.77 \%$ of the price, and the price of $11.23 \%$ needs to be explained by other factors. Table 5 shows the regression effect of the price and search volume regression model by $\mathrm{F}$ saliency statistics. The $\mathrm{P}$ value in the price and search volume analysis is equal to $7.2 \times$ $10^{-83}$, which is much smaller than the significance level of 0.05 , explaining that the regression effect of the regression model on price and search volume is more significant. It can be seen from Table 6 that the $\mathrm{P}$ value of the search volume is $7.27 \times 10^{-83}$, which is much smaller than the significance level of 0.05 , indicating 

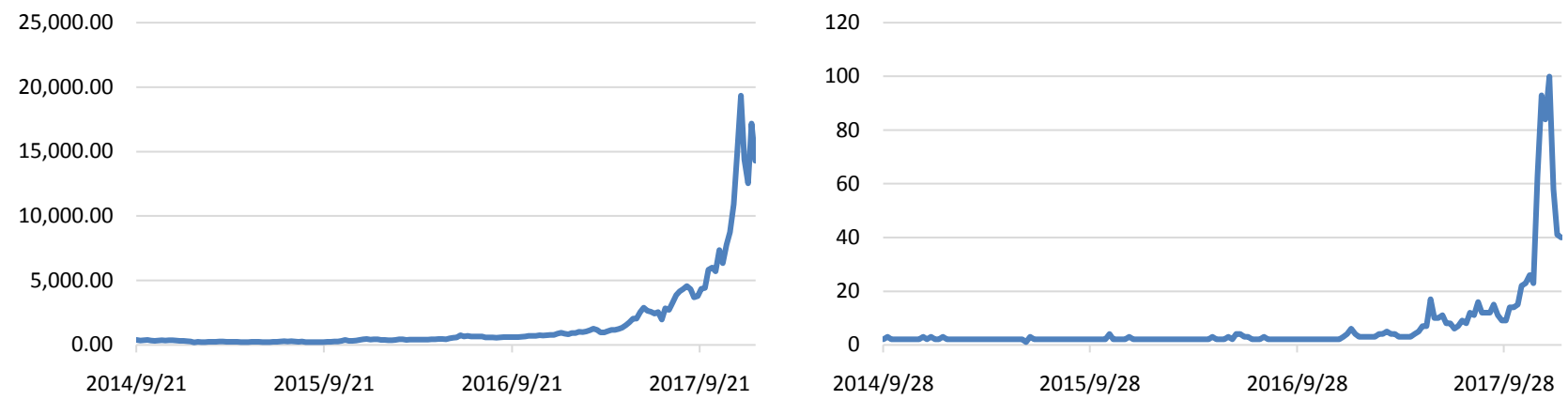

Figure 3. Trends in price and search volume.

Table 4. Regression statistics for price and search.

\begin{tabular}{cc}
\hline & Regression statistics \\
\hline Multiple R & 0.942552 \\
R Square & 0.888404 \\
Adjusted R square & 0.887747 \\
Standard error & 1104.626 \\
Observations & 172
\end{tabular}

Table 5. Regression statistics for price and search.

\begin{tabular}{cccccc}
\hline & $\mathrm{df}$ & $\mathrm{SS}$ & $\mathrm{MS}$ & $\mathrm{F}$ & Significance $\mathrm{F}$ \\
\hline Regression analysis & 1 & $1.65 \mathrm{E}+09$ & $1.65 \mathrm{E}+09$ & 1353.4 & $7.27 \mathrm{E}-83$ \\
residual & 170 & $2.1 \mathrm{E}+08$ & 1220198 & & \\
total & 171 & $1.86 \mathrm{E}+09$ & & & \\
\hline
\end{tabular}

Table 6. Variance regression parameters for price and search volume.

\begin{tabular}{ccccccc}
\hline & Cofficients & Standard error & $\mathrm{t}$ Stat & P value & Lower 95\% & Upper 95\% \\
\hline Intercept & 76.21922 & 95.4564 & 0.7985 & 0.425711 & -112.213 & 246.6516 \\
XVariable & 210.2274 & 5.714584 & 36.78788 & $7.27 \mathrm{E}-83$ & $1.99 \mathrm{E}+02$ & 221.5081 \\
\hline
\end{tabular}

that the regression coefficient of the search volume is significant, and the search volume has a correlation with the price.

From the above regression analysis, the search volume has a significant linear relationship with the price. Because the amount of search is related to the user, according to Metcalf's law, the network value is proportional to the square of the number of users participating in the network. We make assumptions to test the regression between the square of the search volume and the price of the bitcoin blockchain network. As shown in Figure 4, the coefficient of $\mathrm{R}^{2}=0.598$ is not good for the estimation model. Therefore, the linear relationship between search volume and price is more obvious.

\subsection{Multivariate Analysis of Digital Asset Valuation Regression}

According to the above single variable analysis, we use the mining difficulty and 
search volume based on the POW mechanism as the independent variable, the mining difficulty squared and the search volume as the detection factor, and the bitcoin blockchain digital asset price as the dependent variable for multivariate Regression fitting analysis. It can be seen from Table 7 that Multiple $\mathrm{R}$ is equal to 0.9839 , indicating that the correlation between the independent variable and the dependent variable is high and positively correlated. $\mathrm{R}$ Square is equal to 0.9681 , and the square of the mining difficulty is highly matched to the price of the search volume. Adjusted R Square has a value equal to 0.9677 , indicating that the two independent variables can account for $96.77 \%$ of the bitcoin price, and $3.23 \%$ is explained by other factors. Table 8 uses Significance F to determine the regression effect of the multivariate regression model. The $\mathrm{P}$ value of the analysis is equal to $4.2 \times 10^{-127}$, which is much smaller than the significance level of 0.05 , indicating that the regression effect of the multivariate regression model is more significant. It can be seen from Table 9 that the $\mathrm{P}$ value of the square of difficulty in

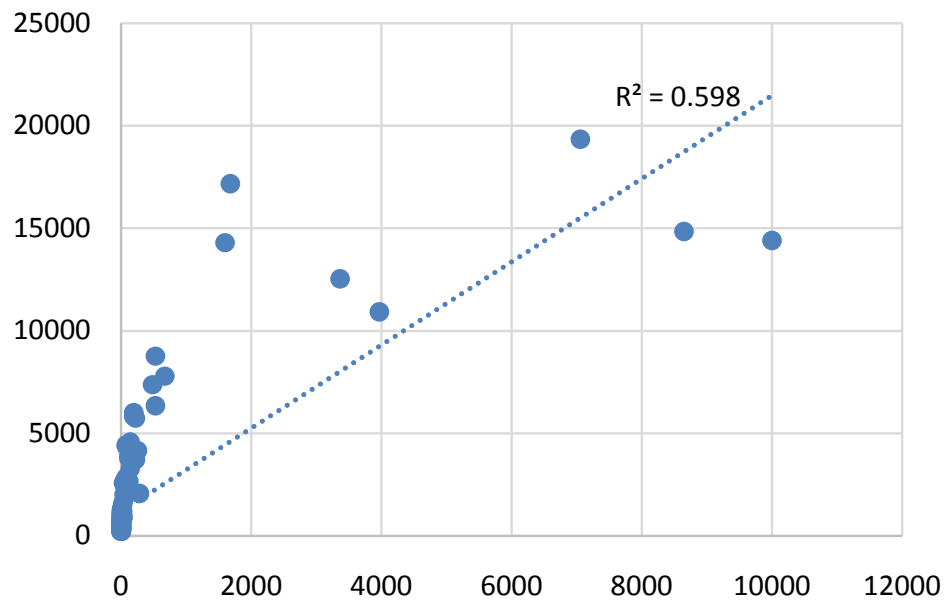

Figure 4. Scatter plot of prices and search volume squared.

Table 7. Multivariate regression statistics.

\begin{tabular}{cc}
\hline & Regression statistics \\
\hline Multiple R & 0.983899 \\
R Square & 0.968057 \\
Adjusted R square & 0.967679 \\
Standard error & 592.7365 \\
Observations & 172 \\
\hline
\end{tabular}

Table 8. Multivariate variance analysis table.

\begin{tabular}{cccccc}
\hline & df & SS & MS & F & Significance F \\
\hline Regression analysis & 2 & $1.80 \mathrm{E}+09$ & $9.0 \mathrm{E}+08$ & 2560.81 & $4.20 \mathrm{E}-127$ \\
Residual & 169 & 5937588 & 351336.5 & & \\
Total & 171 & $1.86 \mathrm{E}+09$ & & & \\
\hline
\end{tabular}


Table 9. Multivariate variance analysis table.

\begin{tabular}{ccccccc}
\hline & Cofficients & Standard error & $\mathrm{t}$ Stat & P value & Lower 95\% & Upper 95\% \\
\hline Intercept & 81.36343 & 51.2201 & 1.588447 & 0.114054 & -19.745 & 182.4084 \\
$X$ Variable1 & $2.72 \mathrm{E}-21$ & $1.32 \mathrm{E}-22$ & 20.52834 & $8.98 \mathrm{E}-48$ & $2.46 \mathrm{E}-21$ & $2.98 \mathrm{E}-21$ \\
$X$ Variable2 & 106.1085 & 5.926864 & 17.90297 & $7.06 \mathrm{E}-41$ & 94.40823 & 117.8087 \\
\hline
\end{tabular}

mining calculation is $8.98 \times 10^{-48}$, and the $\mathrm{P}$ value of the search quantity is $7.06 \times$ $10^{-41}$ which is much smaller than the significance level of 0.05 , indicating that the regression coefficients of the two independent variables are significant.

In summary, we use the mining difficulty $x_{1}$ and the search volume $x_{2}$ as the independent variables, and the bitcoin blockchain digital asset price $Y$ as the dependent variable to obtain the multivariate regression linear equation, which is:

$$
Y=2.72 * 10^{-21} x_{1}^{2}+106.1085 * x_{2}+81.3634+\mu
$$

We add a random perturbation term $\mu$ to the regression equation, where the random disturbance term includes the influence of the neglected factors in the two independent variables of the mining calculation difficulty and the search volume, the observation error influence of the two independent variable observations, and the model relationship setting error, and impact of the effects and other random factors.

\section{Conclusions}

In summary, for the analysis of blockchain digital asset price estimates, we should combine the "human behavior" and "positivism" methods, first of all, based on the mainstream news media reports to analyze the main basis of the impact of blockchain digital asset prices, including safety events, national policies, market recognition, halving of mining cycles, forks and other digital assets, and then based on the Proof of Work (POW) mechanism, using mining calculation difficulty and keyword search variable. According to the analysis method proposed in the paper, the price of the blockchain digital assets is predicted and judged, and the price trend of the blockchain digital assets is comprehensively analyzed, and rational investment is made.

The value of the traditional legal currency comes from the country's credit endorsement, which solves the problem of mistrust through national credit. However, due to its decentralization mechanism, blockchain digital assets do not have third party credit endorsements, but their value does exist. Firstly, the basic value of blockchain digital assets based on POW mechanism comes from its mining value, including mining machine input cost, power consumption and labor cost. Secondly, taking gold as an example, in addition to its basic cost value, the value of gold is based on the public's consensus on the recognition of gold, the value of digital assets, along with the gradual growth of the blockchain digital asset market and the gradual formation of user systems. The consensus value of blockchain digital assets has gradually deepened. 


\section{Conflicts of Interest}

The authors declare no conflicts of interest regarding the publication of this paper.

\section{References}

[1] Nakamoto, A. (2008) Bitcoin: A Peer-to-Peer Electronic Cash System.

[2] Conrad, C., Custovic, A. and Ghysels, E. (2018) Long- and Short-Term Cryptocurrency Volatility Components: A GARCH-MIDAS Analysis. Social Science Electronic Publishing.

[3] Hong, K.H. (2017) Bitcoin as an Alternative Investment Vehicle. Information Technology \& Management, 18, 265-275. https://doi.org/10.1007/s10799-016-0264-6

[4] Cocco, L. and Marchesi, M. (2016) Modeling and Simulation of the Economics of Mining in the Bitcoin Market. PLOS ONE, 11, e0164603. https://doi.org/10.1371/journal.pone.0164603

[5] Jang, H. and Lee, J. (2018) An Empirical Study on Modeling and Prediction of Bitcoin Prices with Bayesian Neural Networks Based on Blockchain Information. IEEE Access, 6, 5427-5437. https://doi.org/10.1109/ACCESS.2017.2779181

[6] Iwamura, M., Kitamura, Y., Matsumoto, T., et al. (2014) Can We Stabilize the Price of a Cryptocurrency? Understanding the Design of Bitcoin and Its Potential to Compete with Central Bank Money. Discussion Paper.

[7] Takaishi, T. (2018) Statistical Properties and Multifractality of Bitcoin. Physica A: Statistical Mechanics and its Applications, 506, 507-519.

https://doi.org/10.1016/j.physa.2018.04.046

[8] Kristoufek, L. (2018) On Bitcoin Markets (in)Efficiency and its Evolution. Physica A: Statistical Mechanics and its Applications, 503, 257-262.

https://doi.org/10.1016/j.physa.2018.02.161

[9] Vitt, D.C. (2013) Breaking Bitcoin: Does Cryptocurrency Exchange Activity Lead to Increased Real Activity Outside Cryptocurrency Exchanges? SSRN Electronic Journal.

[10] Palamara, E., Zacharias, N., Xanthopoulou, M., et al. (2016) Technology Issues of Byzantine Glazed Pottery from Corinth, Greece. Microchemical Journal, 129, 137-150. https://doi.org/10.1016/j.microc.2016.06.008

[11] Bentov, I., Lee, C., Mizrahi, A., et al. (2014) Proof of Activity: Extending Bitcoin's Proof of Work via Proof of Stake.

[12] Zhang, R. and Preneel, B. (2017) On the Necessity of a Prescribed Block Validity Consensus: Analyzing Bitcoin Unlimited Mining Protocol. International Conference.

[13] Huberman, G., Leshno, J.D. and Moallemi, C.C. (2017) Monopoly without a Monopolist: An Economic Analysis of the Bitcoin Payment System. Columbia Business School Research Paper No. 17-92. https://doi.org/10.2139/ssrn.3025604

[14] Hornberger, J. and Hirth, R.A. (2012) Financial Implications of Choice of Dialysis Type of the Revised Medicare Payment System: An Economic Analysis. American Journal of Kidney Diseases, 60, 280-287. https://doi.org/10.1053/j.ajkd.2012.03.010

[15] Katsiampa, P. (2017) Volatility Estimation for Bitcoin: A Comparison of GARCH Models. Economics Letters, 158, 3-6. https://doi.org/10.1016/j.econlet.2017.06.023

[16] Narayanan, A., Bonneau, J., Felten, E., Miller, A. and Goldfeder, S. (2016) Bitcoin 
and Cryptocurrency Technologies: A Comprehensive Introduction. Princeton University Press, Princeton.

[17] Elbahrawy, A., Alessandretti, L., Kandler, A., Pastor-Satorras, R. and Baronchelli, A. (2017) Evolutionary Dynamics of the Cryptocurrency Market. Royal Society Open Science, 4, Article ID: 170623. https://doi.org/10.1098/rsos.170623

[18] Bianchetti, M., Ricci, C. and Scaringi, M. (2018) Are Cryptocurrencies Real Financial Bubbles? Evidence from Quantitative Analyses. Social Science Electronic Publishing, Rochester.

[19] Thrane, C. (2015) On the Relationship between Length of Stay and Total Trip Expenditures: A Case Study of Instrumental Variable (IV) Regression Analysis. Tourism Economics, 21, 357-367. https://doi.org/10.5367/te.2014.0357

[20] Wang, J., Huang, D., Chang, S.C. and Brant, L. (2013) On-Site Earthquake Early Warning with Multiple Regression Analysis: Featuring Two User-Friendly Applications for Excel. Computers \& Geosciences, 58, 1-7. https://doi.org/10.1016/j.cageo.2013.04.018

[21] Balcilar, M., Demirer, R., Gupta, R. and Wohar, M.E. (2017) Differences of Opinion and Stock Market Volatility: Evidence from a Nonparametric Causality-in-Quantiles Approach. Journal of Economics and Finance, 42, 339-351. https://doi.org/10.1007/s12197-017-9404-Z

[22] Hayes, A. (2015) A Cost of Production Model for Bitcoin. Social Science Electronic Publishing, Rochester. 